\title{
Convergence Analysis on Stochastic Collocation Methods for the Linear Schrödinger Equation with Random Inputs
}

\author{
Zhizhang Wu and Zhongyi Huang* \\ Department of Mathematical Sciences, Tsinghua University, Beijing 100084, China
}

Received 7 January 2019; Accepted (in revised version) 11 March 2019

\begin{abstract}
In this paper, we analyse the stochastic collocation method for a linear Schrödinger equation with random inputs, where the randomness appears in the potential and initial data and is assumed to be dependent on a random variable. We focus on the convergence rate with respect to the number of collocation points. Based on the interpolation theories, the convergence rate depends on the regularity of the solution with respect to the random variable. Hence, we investigate the dependence of the stochastic regularity of the solution on that of the random potential and initial data. We provide sufficient conditions on the random potential and initial data to ensure the smoothness of the solution and the spectral convergence. Finally, numerical results are presented to support our analysis.
\end{abstract}

AMS subject classifications: 65M12, 81Q05, 60H25

Key words: Schrödinger equation, stochastic collocation methods, convergence analysis, uncertainty quantification.

\section{Introduction}

The linear Schrödinger equation describes the motion of electrons in the external field, which has been studied both theoretically $[17,39]$ and numerically $[8,19]$. Recently, the linear Schrödinger equation with a random potential $[1,30]$ has attracted a great deal of attention. The randomness may arise from disordered structures like amorphous solids, random alloys and non-crystalline systems, thermal fluctuation or randomly distributed impurities $[2,12,13,15,21,22,25]$. The random Schrödinger operators have been intensively studied in a theoretical way $[5,6,14,23,24]$. In addition, Schrödinger equations with random initial data are investigated recently [26]. However, the numerical literatures on

*Corresponding author.

Emails: wzz14@mails.tsinghua.edu.cn (Z.Z.Wu), zhuang@math.tsinghua.edu.cn (Z. Y. Huang) 
them are not so abundant [7]. Indeed, this kind of problems belongs to uncertainty quantification (UQ) for PDEs with random inputs, where randomness could appear in initial conditions, boundary conditions, coefficients of the equations, etc. There have been quite a few numerical methods developed for UQ in recent years. Among them, the stochastic collocation method and the stochastic Galerkin method [28, 34-38] are two of most popular methods, both of which make use of the polynomial approximation theory. They have been successfully applied to many physical and engineering problems $[16,18,20,31,40]$. Convergence analyses on both methods for different kinds of differential equations have also been established [3, 4, 27, 29, 32, 41]. For the linear Schrödinger equation, $\mathrm{Wu}$ and Huang applied the stochastic Galerkin method to the Schrödinger equation with a periodic potential and a random external potential [33]. Nevertheless, to the best of our knowledge, there is not any convergence result on both methods for the linear Schrödinger equation with a random potential.

In this paper, we focus on the convergence analysis on the stochastic collocation method for the Schrödinger equation with a random potential and random initial data. Since the stochastic collocation method makes use of the polynomial approximation theory, the convergence rate of the stochastic method w.r.t. the number of collocation points relies on the regularity of the solution w.r.t. the random variable, which in turn depends on the smoothness of the potential and initial data w.r.t. the random variable. We first study the stochastic regularity for the Schrödinger equation where the spatial variable $x \in \mathbb{R}^{d}$. To study the stochastic regularity, we will make use of some well-posedness results on the Schrödinger equation in the deterministic case, for which there have been numerous results [17,39]. The results given by Yajima [39] will be fully utilized. The well-posedness of the random Schrödinger equation will be established and two cases of sufficient conditions to ensure the stochastic regularity will be presented. As a usual practice in numerical computations for the Schrödinger equation, we compute it in a truncated domain. Hence we then consider the Schrödinger equation in a bounded domain and provide sufficient conditions on the random potential and initial data to ensure the spectral convergence of the stochastic collocations. The more smoothly the potential and initial data depend on the random variable, the faster the convergence rate will be. It turns out that the stochastic collocation method is a solid method when the magnitude of the randomness is within an appropriate range.

The outline of this paper is as follows. In Section 2, the well-posedness result in the deterministic case by Yajima is reviewed. In Section 3, we study the stochastic regularity of the random linear Schrödinger equation. Then the time splitting based stochastic collocation method and its corresponding convergence results are presented in Section 4 . We give some numerical results in Section 5 to support our analyses. Finally, a conclusion is given in Section 6.

Throughout this paper, the analysis will be performed for the case where the random variable $y \in \mathbb{R}$ for simplicity. However, similar analyses can be extended to the highdimensional cases using tensor-product rule in the parametric space, see e.g., [3]. 


\section{Well-posedness in the deterministic case}

In this section, we will review some results on the well-posedness of the solution to a deterministic linear Schrödinger equation presented by Yajima, which are inspiring for our subsequent analysis. For detailed proofs of them, please refer to [39].

Consider the initial value problem for the time dependent Schrödinger equation:

$$
\left\{\begin{array}{l}
i \frac{\partial u}{\partial t}=-\frac{1}{2} \Delta_{x} u+V(t, x) u, \quad t \in[-T, T]=I_{T}, \quad x \in \mathbb{R}^{n} \\
\left.u\right|_{t=s}=u_{0}(x)
\end{array}\right.
$$

where $V(t, x)$ is a real-valued function.

By Duhamel's principle, the solution of (2.1) is related to the following integral equation:

$$
u(t)=U_{0}(t-s) u_{0}-i \int_{s}^{t} U_{0}(t-\tau) V(\tau) u(\tau) d \tau,
$$

where $U_{0}(t)=\exp (i t \Delta / 2)$ is the free propagator and $V(t)$ is the multiplication operator by $V(t, x)$. For an interval $I$ and $m, r \geq 1$, we denote by $L^{m, r}(I)$ the Banach space of $L^{m}\left(\mathbb{R}^{n}\right)$ valued $r$-summable functions over $I$ :

$$
L^{m, r}(I)=\left\{u:\left[\int_{I}\left(\int_{\mathbb{R}^{n}}|u(t, x)|^{m} d x\right)^{r / m} d t\right]^{1 / r}=\|u\|_{m, r}<\infty\right\} .
$$

For real numbers $p \geq 1, \alpha \geq 1, \beta>1, T>0$ satisfying $0 \leq 1 / \alpha<1-n / 2 p$, we introduce the following space:

$$
\mathcal{M}(p, \alpha, \beta, T)=L^{p, \alpha}\left(I_{T}\right)+L^{\infty, \beta}\left(I_{T}\right) .
$$

We say $V \in \mathcal{M}(p, \alpha, \beta, T)$ if there exists $V_{1} \in L^{p, \alpha}\left(I_{T}\right), V_{2} \in L^{\infty, \beta}\left(I_{T}\right)$ such that $V=V_{1}+V_{2}$. The norm of $\mathcal{M}(p, \alpha, \beta, T)$ is defined as

$$
\|V\|_{\mathcal{M}(p, \alpha, \beta, T)}=\inf \left\{\left\|V_{1}\right\|_{p, \alpha}+\left\|V_{2}\right\|_{\infty, \beta}: V=V_{1}+V_{2}\right\} .
$$

Then we have the following theorem:

Theorem 2.1. If $V \in \mathcal{M}(p, \alpha, \beta, T)$ and $q=\frac{2 p}{p-1}, \theta=4 p / n$. Then:

1. Eq. (2.2) has a unique solution $u \in C\left(I_{T}, L^{2}\left(\mathbb{R}^{n}\right)\right) \cap L^{q, \theta}\left(I_{T}\right)$, for every $u_{0} \in L^{2}\left(\mathbb{R}^{n}\right)$ and $s \in I_{T}$.

2. $\|u(t)\|_{2}=\left\|u_{0}\right\|_{2}, \forall t \in I_{T}$.

The proof of this theorem can be found on pp. 419-424 of [39]. In addition, the proof relies on the lemmas we will subsequently introduce. 
By $Q$ and $S$ we denote the integral operators

$$
\begin{aligned}
& (Q u)(t)=\int_{0}^{t} U_{0}(t-s) V(s) u(s) d s, \\
& (S u)(t)=\int_{0}^{t} U_{0}(t-s) u(s) d s .
\end{aligned}
$$

Then

$$
(Q u)(t)=(S V u)(t) .
$$

And we introduce the following Banach spaces. Let $I=[-a, a]$ and $\ell$ be a parameter with $0 \leq n(1 / 2-1 / \ell)<1$ :

$$
\begin{aligned}
& \mathcal{H}(a, \ell)=C\left(I, L^{2}\left(\mathbb{R}^{n}\right)\right) \bigcap L^{\ell, \theta}(I), \quad \theta=\frac{4 \ell}{n(\ell-2)}, \\
& \mathcal{H}^{*}(a, \ell)=L^{1}\left(I, L^{2}\left(\mathbb{R}^{n}\right)\right)+L^{\ell^{\prime}, \theta^{\prime}}(I), \quad \ell^{\prime}=\frac{\ell}{\ell-1}, \quad \theta^{\prime}=\frac{\theta}{\theta-1}, \\
& \mathcal{Y}(a, \ell)=\left\{u: u \in C\left(I, H^{2}\left(\mathbb{R}^{n}\right)\right), \quad \dot{u} \in \mathcal{H}(a, \ell)\right\}, \quad \dot{u}=\partial u / \partial t, \\
& \mathcal{Y}^{*}(a, \ell)=\left\{u: u \in C\left(I, L^{2}\left(\mathbb{R}^{n}\right)\right), \dot{u} \in \mathcal{H}^{*}(a, \ell)\right\},
\end{aligned}
$$

with the norms

$$
\begin{aligned}
& \|u\|_{\mathcal{H}(a, \ell)}=\|u\|_{2, \infty}+\|u\|_{\ell, \theta}, \\
& \|u\|_{\mathcal{H}^{*}(a, \ell)}=\inf \left\{\left\|u_{1}\right\|_{2,1}+\left\|u_{2}\right\|_{\ell^{\prime}, \theta^{\prime}}: u=u_{1}+u_{2}\right\}, \\
& \|u\|_{\mathcal{Y}(a, \ell)}=\sup _{t \in I}\|u(t)\|_{H^{2}}+\|\dot{u}\|_{\mathcal{H}(a, \ell)} \\
& \|u\|_{\mathcal{Y}^{*}(a, \ell)}=\|u(t)\|_{2, \infty}+\|\dot{u}\|_{\mathcal{H}^{*}(a, \ell)} .
\end{aligned}
$$

Then we have a lemma for the free propagator $U_{0}$ and the integral operator $S$.

Lemma 2.1. Let $0 \leq n(1 / 2-1 / \ell)<1$. Then

$$
\begin{array}{ll}
\left\|U_{0}(\cdot) f\right\|_{\mathcal{H}(a, \ell)} \leq C\|f\|_{2,} & f \in L^{2}\left(\mathbb{R}^{n}\right), \\
\|S u\|_{\mathcal{H}(a, \ell)} \leq C\|u\|_{\mathcal{H}^{*}(a, \ell)}, & u \in \mathcal{H}^{*}(a, \ell), \\
\|S u\|_{\mathcal{Y}(a, \ell)} \leq C(1+a)\|u\|_{\mathcal{Y}^{*}(a, \ell),} & u \in \mathcal{Y}^{*}(a, \ell) .
\end{array}
$$

Here the constant $C>0$ is independent of $a, u$ and $f$.

The proof of this lemma is presented on pp. 419-420 in [39].

Let $q=\frac{2 p}{p-1}$ and

$$
\mathcal{H}(a)=\mathcal{H}(a, q), \quad \mathcal{H}^{*}(a)=\mathcal{H}^{*}(a, q), \quad \mathcal{Y}(a)=\mathcal{Y}(a, q), \quad \mathcal{Y}^{*}(a)=\mathcal{Y}^{*}(a, q) .
$$


Lemma 2.2. Let $V \in \mathcal{M}(p, \alpha, \beta, a)$. Then

$$
\|V u\|_{\mathcal{H}^{*}(a)} \leq(2 a)^{\gamma}\|V\|_{\mathcal{M}}\|u\|_{\mathcal{H}(a), \quad 2 a<1,}
$$

with $\gamma=\min (1-1 / \beta, 1-n / 2 p-1 / \alpha)$.

The proof of this lemma is proposed on pp. 420-421 in [39].

Lemma 2.3. Suppose $V_{\varepsilon} \in \mathcal{M}(p, \alpha, \beta, T)(\varepsilon>0)$ and that

$$
\lim _{\varepsilon \rightarrow 0}\left\|V_{\varepsilon}-V\right\|_{\mathcal{M}(p, \alpha, \beta, T)}=0 .
$$

Suppose also that

$$
\lim _{\varepsilon \rightarrow 0}\left\|u_{0 \varepsilon}-u_{0}\right\|_{2}=0 .
$$

Let $u_{\varepsilon} \in \mathcal{H}(T)$ and $u \in \mathcal{H}(T)$ be the solutions of

$$
u_{\varepsilon}(t)=U_{0}(t-s) u_{0 \varepsilon}-i \int_{s}^{t} U_{0}(t-\tau) V_{\varepsilon}(\tau) u_{\varepsilon}(\tau) d \tau,
$$

and (2.2) respectively. Then

$$
\lim _{\varepsilon \rightarrow 0}\left\|u_{\varepsilon}-u\right\|_{\mathcal{H}(T)}=0 .
$$

The proof of this lemma is on pp. 422-423 in [39].

Lemma 2.4. Suppose that $u \in \mathcal{H}(T)$ satisfies

$$
u(t)=U_{0}(t) u_{0}-i S V u(t), \quad u_{0} \in H^{2}\left(\mathbb{R}^{n}\right),
$$

and $V u \in \mathcal{Y}^{*}(T)$. Then $u \in \mathcal{Y}(T)$ and it satisfies (2.1) and

$$
\frac{d}{d t} S f(t)=U_{0}(t) f(0)+S \dot{f}(t), \quad f=V u .
$$

The proof of this lemma can be seen on pp. 421 in [39].

Remark 2.1. The proof of Lemma 2.4 is based on the fact that $U_{0}(t) u_{0} \in \mathcal{Y}(T)$ for $u_{0} \in$ $H^{2}\left(\mathbb{R}^{n}\right)$ and

$$
\left(-i \frac{d}{d t}+H_{0}\right) S f(t)=-i f(t), \quad f \in C^{\infty}\left(I_{1}, C_{0}^{\infty}\left(\mathbb{R}^{n}\right)\right),
$$

where $I_{1}=(-a, a)$ and $H_{0}=-\frac{1}{2} \Delta_{x}$.

Lemma 2.5. Let $V \in C^{1}\left(I_{T}, L^{\infty}\left(\mathbb{R}^{n}\right)\right)$ and $u_{0} \in H^{2}\left(\mathbb{R}^{n}\right)$. Then the solution $u \in \mathcal{H}(T)$ of (2.2) belongs to $\mathcal{Y}(T)$ and it satisfies (2.1),

$$
i \dot{u}(t)=H(t) u(t), \quad H(t)=H_{0}+V(t)=-\frac{1}{2} \Delta_{x}+V(t), \quad \dot{u}=\partial u / \partial t .
$$

The proof of this lemma is presented on pp. 423-424 in [39]. 


\section{Schrödinger equation with a random potential}

We consider the linear Schrödinger equation with a random potential and random initial data

$$
\left\{\begin{array}{l}
i \frac{\partial u}{\partial t}=-\frac{1}{2} \Delta_{x} u+V(t, x, y) u, \quad t \in I_{T}=[-T, T], \quad x \in \mathbb{R}^{n}, \quad y \in \Gamma, \\
\left.u\right|_{t=s}=u_{0}(x, y)
\end{array}\right.
$$

where $y$ is a random variable with a density function $\rho(y)$. And we assume $\Gamma \subset \mathbb{R}^{1}$.

By Duhamel's principle, we consider the following integral equation: for $\rho$-a.e. $y \in \Gamma$,

$$
u(t, y)=U_{0}(t-s) u_{0}(y)-i \int_{s}^{t} U_{0}(t-\tau) V(\tau, y) u(\tau, y) d \tau .
$$

We also define space

$$
L_{\rho}^{p}(\Gamma, H)=\left\{u(y):\|u\|_{L_{\rho}^{p}(\Gamma, H)}=\left(\int_{\Gamma}\|u(y)\|_{H}^{p} \rho(y) d y\right)^{1 / p}<\infty\right\},
$$

where $H$ is some function space.

\subsection{Well-posedness}

Theorem 3.1. Assume that for $\rho$-a.e. $y \in \Gamma, V(t, x, y) \in \mathcal{M}(p, \alpha, \beta, T)$ and there exists a constant $C$ such that $\left\|u_{0}(\cdot, y)\right\|_{2} \leq C$. Then for Eq. (3.2), there exists a unique solution $u \in L_{\rho}^{2}\left(\Gamma, L^{\infty}\left(I_{T}, L^{2}\left(\mathbb{R}^{n}\right)\right)\right)$.

Proof. Since $V(t, x, y) \in \mathcal{M}(p, \alpha, \beta, T)$ for $\rho$-a.e. in $y \in \Gamma$, by Theorem 2.1, for $\rho$-a.e. $y \in \Gamma$ there exists a unique $u(\cdot, \cdot, y) \in C\left(I_{T}, L^{2}\left(\mathbb{R}^{n}\right)\right)$ for $u_{0}(\cdot, y) \in L^{2}\left(\mathbb{R}^{n}\right)$. Moreover, $\|u(t, \cdot, y)\|_{2}^{2}=$ $\left\|u_{0}(\cdot, y)\right\|_{2}^{2}$ for any $t \in I_{T}$.

Integrating w.r.t. $\rho(y) d y$, we have

$$
\|u\|_{L_{\rho}^{2}\left(\Gamma, L^{\infty}\left(I_{T}, L^{2}\left(\mathbb{R}^{n}\right)\right)\right)}=\left\|u_{0}\right\|_{L_{\rho}^{2}\left(\Gamma, L^{2}\left(\mathbb{R}^{n}\right)\right)} \leq C .
$$

Finally, it's easy to see that $u \in L_{\rho}^{2}\left(\Gamma, L^{\infty}\left(I_{T}, L^{2}\left(\mathbb{R}^{n}\right)\right)\right)$.

\subsection{Stochastic regularity}

We want to investigate the $H^{k}$-regularity of $u$ w.r.t. $y$. Assume that the weak derivative of $V$ w.r.t $y$ exists. Taking the partial derivative of $u$ w.r.t. $y$ in (3.2), we have

$$
\partial_{y} u(t)=U_{0}(t-s) u_{0 y}-i \int_{s}^{t} U_{0}(t-\tau) V(\tau) \partial_{y} u(\tau) d \tau-i \int_{s}^{t} U_{0}(t-\tau) \partial_{y} V(\tau) u(\tau) d \tau,
$$

where $u_{0 y}=\partial_{y} u_{0}$. We focus here on the case where the starting time $s=0$. 
Remark 3.1. If we take the partial derivative of $u$ w.r.t. $y$ in (3.1), we have

$$
\left\{\begin{array}{l}
i \frac{\partial}{\partial t} \partial_{y} u=-\frac{1}{2} \Delta_{x} \partial_{y} u+V \partial_{y} u+\partial_{y} V u, \quad t \in I_{T}, \quad x \in \mathbb{R}^{n}, \quad y \in \Gamma, \\
\left.\partial_{y} u\right|_{t=s}=\partial_{y} u_{0}(x, y) .
\end{array}\right.
$$

By Duhamel's principle, the integral equation related to Eq. (3.6) is just Eq. (3.5).

\subsubsection{Bounded case}

Theorem 3.2. Assume that there exists a constant $C>0$, such that for $\rho$-a.e. $y \in \Gamma$,

- $V(t, x, y) \in \mathcal{M}(p, \alpha, \beta, T)$

- $\partial_{y} V(t, x, y) \in \mathcal{M}(p, \alpha, \beta, T)$

- $\|V(\cdot, \cdot, y)\|_{\mathcal{M}(p, \alpha, \beta, T)} \leq C$

- $\left\|\partial_{y} V(\cdot, \cdot, y)\right\|_{\mathcal{M}(p, \alpha, \beta, T)} \leq C$

- $\left\|u_{0}(\cdot, y)\right\|_{2} \leq \mathrm{C}$

- $\left\|\partial_{y} u_{0}(\cdot, y)\right\|_{2} \leq C$

Then for Eq. (3.5), there exists a unique solution $\partial_{y} u \in L_{\rho}^{2}\left(\Gamma, L^{\infty}\left(I_{T}, L^{2}\left(\mathbb{R}^{n}\right)\right)\right)$, i.e., $u \in$ $H_{\rho}^{1}\left(\Gamma, L^{\infty}\left(I_{T}, L^{2}\left(\mathbb{R}^{n}\right)\right)\right)$.

Proof. By Theorem 3.1, we have $u \in L_{\rho}^{2}\left(\Gamma, L^{\infty}\left(I_{T}, L^{2}\left(\mathbb{R}^{n}\right)\right)\right)$.

We proceed to show that the $\mathcal{H}(T)$ norm of $u(\cdot, \cdot, y)$ is bounded in $\Gamma$.

The proof is based on that in [39]. For $\rho$-a.e. $y \in \Gamma$, we can construct the global solution $u(\cdot, \cdot, y) \in \mathcal{H}(T)$ in the following way. By Lemma 2.2, if $a$ is sufficiently small, $Q$ is a contraction on $\mathcal{H}(a)$ and hence there exists a local solution $u \in \mathcal{H}(a)$. Then using a standard continuation procedure for the solution of linear integral equations, the global solution $u \in \mathcal{H}(T)$ can be constructed.

Since for $\rho$-a.e.y $\in \Gamma,\|V(\cdot, \cdot, y)\|_{\mathcal{M}(p, \alpha, \beta, T)} \leq C$, there exists a constant $0<A_{1}<\frac{1}{2}$ such that when $\frac{1}{2} A_{1} \leq a \leq A_{1}$,

$$
\begin{array}{ll}
\|Q\|_{\mathcal{B}(\mathcal{H}(a))} \leq \frac{1}{2}, & \rho-\text { a.e. } y \in \Gamma, \\
\left\|(1+i Q)^{-1}\right\|_{\mathcal{B}(\mathcal{H}(a))} \leq 2, & \rho-\text { a.e. } y \in \Gamma,
\end{array}
$$

where $\mathcal{B}(\mathcal{H}(a))$ is the set of bounded linear mapping from $\mathcal{H}(a)$ to itself. By Lemma 2.1 , there exists another constant $A_{2}$ such that $\left\|U_{0}(t) u_{0}(\cdot, y)\right\|_{\mathcal{H}(T)} \leq A_{2}\left\|u_{0}(\cdot, y)\right\|_{2}$ and we 
assume $A_{2}>1$ here without loss of generosity. We choose $a \in\left[\frac{1}{2} A_{1}, A_{1}\right]$ and let $L=\left\lfloor\frac{T}{a}\right\rfloor$. Then for $\rho$-a.e. $y \in \Gamma$,

$$
\begin{aligned}
\|u\|_{\mathcal{H}(T)} \leq & \sum_{j=-L+1}^{L-1}\left\|u I_{B_{j}}(t)\right\|_{\mathcal{H}(T)}+\left\|u I_{[-T,(-L+1) a]}(t)\right\|_{\mathcal{H}(T)} \\
& +\left\|u I_{[(L-1) a, T]}(t)\right\|_{\mathcal{H}(T)} \leq 2 \sum_{j=1}^{L+1} 2^{j} A_{2}^{j}\left\|u_{0}\right\|_{2} \\
\leq C & \left|\frac{2^{L+3} A_{2}^{L+2}-4 A_{2}}{2 A_{2}-1}\right| \leq C\left|\frac{2^{\widetilde{L}+3} A_{2}^{\widetilde{L}+2}-4 A_{2}}{2 A_{2}-1}\right|
\end{aligned}
$$

where $I_{B}$ is the indicator function of set $B, B_{j}=[(j-1) a,(j+1) a], \widetilde{L}=\left\lfloor\frac{2 T}{A_{1}}\right\rfloor$. Take $j>0$ for example, the second inequality above is due to

$$
\begin{aligned}
\left\|u I_{B_{j}}(t)\right\|_{\mathcal{H}(T)} & =\left\|\left(1+i Q^{j a}\right)^{-1} U_{0}(t-j a) u(j a) I_{B_{j}}(t)\right\|_{\mathcal{H}(T)} \\
& \left.\leq 2 \| U_{0}(t-j a) u(j a) I_{B_{j}}(t)\right) \|_{\mathcal{H}(T)} \\
& \leq 2 A_{2}\|u(j a)\|_{2} \leq 2 A_{2}\left\|u I_{B_{j-1}}(t)\right\|_{\mathcal{H}(T)} \\
& \leq \cdots \leq 2^{j+1} A_{2}^{j+1}\left\|u_{0}\right\|_{2},
\end{aligned}
$$

where $Q^{j a}$ is a shifted operator of $Q, Q^{j a} u(t)=\int_{j a}^{t} U_{0}(t-\tau) V(\tau) u(\tau) d \tau$. Hence the $\mathcal{H}(T)$ norm of $u(\cdot, \cdot, y)$ is bounded in $\Gamma$.

For $\partial_{y} u$, by Lemmas 2.1 and 2.2, since we have $\partial_{y} u_{0} \in L^{2}\left(\mathbb{R}^{n}\right)$ and $\partial_{y} V(t, x, y) \in$ $\mathcal{M}(p, \alpha, \beta, T)$ for $\rho$-a.e. $y \in \Gamma$, then $U_{0}(t) \partial_{y} u_{0} \in \mathcal{H}(T)$ and $\left(Q_{y} u\right)(t) \in \mathcal{H}(T)$. Then using the same continuation procedure, we can construct the unique solution of Eq. (3.5), $\partial_{y} u(\cdot, \cdot, y) \in \mathcal{H}(T)$, a.e. $y \in \Gamma$.

Since for $\rho$-a.e. $y \in \Gamma,\left\|\partial_{y} V(\cdot, \cdot, y)\right\|_{\mathcal{M}(p, \alpha, \beta, T)} \leq C$ and $\left\|\partial_{y} u_{0}(\cdot, y)\right\|_{2} \leq C$, then $\left\|U_{0}(t) \partial_{y} u_{0}\right\|_{\mathcal{H}(T)}$ and $\left\|\left(S \partial_{y} V u\right)(t)\right\|_{\mathcal{H}(T)}$ is bounded in $\Gamma$. Repeating the above proof, we know that the $\mathcal{H}(T)$ norm of $\partial_{y} u$ is bounded in $\Gamma$.

In particular, $u \in H_{\rho}^{1}\left(\Gamma, L^{\infty}\left(I_{T}, L^{2}\left(\mathbb{R}^{n}\right)\right)\right)$.

For $\partial_{y}^{k} u$, from Eq. (3.2), we have

$$
\partial_{y}^{k} u(t)=U_{0}(t-s) \partial_{y}^{k} u_{0}-i \int_{0}^{t} U_{0}(t-\tau) V(\tau) \partial_{y}^{k} u(\tau) d \tau-i \int_{s}^{t} U_{0}(t-\tau) f(\tau) d \tau,
$$

where $f=\sum_{j=1}^{k}\left(\begin{array}{l}k \\ j\end{array}\right) \partial_{y}^{j} V \partial_{y}^{k-j} u$. For stochastic regularity of higher order, we have

Theorem 3.3. Assume that there exists a constant $C>0$ and for $\rho$-a.e. $y \in \Gamma$,

- $V(t, x, y) \in \mathcal{M}(p, \alpha, \beta, T)$

- $\partial_{y}^{j} V(t, x, y) \in \mathcal{M}(p, \alpha, \beta, T), j=1, \cdots, k$ 
- $\|V(\cdot, \cdot, y)\|_{\mathcal{M}(p, \alpha, \beta, T)} \leq \mathrm{C}$

- $\left\|\partial_{y}^{j} V(\cdot, \cdot, y)\right\|_{\mathcal{M}(p, \alpha, \beta, T)} \leq C, j=1, \cdots, k$

- $\left\|\partial_{y}^{j} u_{0}(\cdot, y)\right\|_{2} \leq C, j=0,1, \cdots, k$

Then $u \in H_{\rho}^{k}\left(\Gamma, L^{\infty}\left(I_{T}, L^{2}\left(\mathbb{R}^{n}\right)\right)\right)$.

This theorem can be proved using induction.

Remark 3.2. In Theorem 3.3, we would prove an upper bound $C(T, k)$ on the $H^{k}$-norm of $u$ using induction. The upper bound $C(T, k)$ is dependent on $k$ and $T$. It could blow up as $k \rightarrow \infty$. And it could also blow up as $T \rightarrow \infty$.

\subsubsection{Singular case}

We can see that in the proof of Theorem 3.1, we have $u \in L_{\rho}^{\infty}\left(\Gamma, L^{\infty}\left(I_{T}, L^{2}\left(\mathbb{R}^{n}\right)\right)\right)$ and in that of Theorem 3.2, we have the $\mathcal{H}(T)$-norm of $u$ is bounded in $\Gamma$. Thus it could admit that $V$ has singularities in $y$. However, it turns out that we strengthen the space-time regularity to ensure the well-posedness of (3.5). We first need the following lemmas which are analogous to Lemmas 2.3 and 2.5 respectively:

Lemma 3.1. Suppose for $\rho$-a.e. $y \in \Gamma, V_{\varepsilon}, V_{y \varepsilon} \in \mathcal{M}(p, \alpha, \beta, T)(\varepsilon>0)$ and

$$
\begin{aligned}
& \lim _{\varepsilon \rightarrow 0}\left\|V_{\mathcal{\varepsilon}}(\cdot, \cdot, y)-V(\cdot, \cdot, y)\right\|_{\mathcal{M}(p, \alpha, \beta, T)}=0, \\
& \lim _{\varepsilon \rightarrow 0}\left\|V_{y \mathcal{\varepsilon}}(\cdot, \cdot, y)-\frac{\partial V}{\partial y}(\cdot, \cdot, y)\right\|_{\mathcal{M}(p, \alpha, \beta, T)}=0 .
\end{aligned}
$$

Suppose also that

$$
\begin{aligned}
& \lim _{\varepsilon \rightarrow 0}\left\|u_{0 \varepsilon}(\cdot, y)-u_{0}(\cdot, y)\right\|_{2}=0, \\
& \lim _{\varepsilon \rightarrow 0}\left\|\frac{\partial u_{0 \varepsilon}}{\partial y}(\cdot, y)-\frac{\partial u_{0}}{\partial y}(\cdot, y)\right\|_{2}=0 .
\end{aligned}
$$

Let $u_{\mathcal{\varepsilon}}(\cdot, \cdot, y) \in \mathcal{H}(T)$ and $u(\cdot, \cdot, y) \in \mathcal{H}(T)$ be the solutions of

$$
u_{\varepsilon}(t)=U_{0}(t-s) u_{0 \varepsilon}-i \int_{s}^{t} U_{0}(t-\tau) V_{\varepsilon}(\tau) u_{\varepsilon}(\tau) d \tau
$$

and (3.2) respectively. Moreover, let $w_{\mathcal{\varepsilon}}(\cdot, \cdot, y) \in \mathcal{H}(T)$ and $\partial_{y} u(\cdot, \cdot, y) \in \mathcal{H}(T)$ be the solutions of

$$
\begin{gathered}
w_{\varepsilon}(t)=U_{0}(t-s) \partial_{y} u_{0 \varepsilon}-i \int_{s}^{t} U_{0}(t-\tau) V_{\varepsilon}(\tau) w_{\varepsilon}(\tau) d \tau \\
-i \int_{s}^{t} U_{0}(t-\tau) V_{y \varepsilon}(\tau) u_{\varepsilon}(\tau) d \tau
\end{gathered}
$$

and (3.5) respectively. Then a.s.

$$
\lim _{\varepsilon \rightarrow 0}\left\|w_{\varepsilon}(\cdot, \cdot, y)-\partial_{y} u(\cdot, \cdot, y)\right\|_{\mathcal{H}(T)}=0 .
$$


Proof. It's sufficient to consider the case where $s=0$ and $T>0$ small for $\rho$-a.e. $y \in \Gamma$.

By Lemma 2.3,

$$
\lim _{\varepsilon \rightarrow 0}\left\|u_{\varepsilon}(\cdot, \cdot, y)-u(\cdot, \cdot, y)\right\|_{\mathcal{H}(T)}=0 .
$$

Now we denote $Q_{\varepsilon}=S V_{\varepsilon}$ and $Q_{y \varepsilon}=S V_{y \varepsilon}$. By Lemma 2.2, it's easy to see that almost surely

$$
\begin{aligned}
& \lim _{\varepsilon \rightarrow 0}\left\|Q_{\varepsilon}-Q\right\|_{\mathcal{B}(\mathcal{H}(T))}=0, \\
& \lim _{\varepsilon \rightarrow 0}\left\|Q_{y \varepsilon}-Q_{y}\right\|_{\mathcal{B}(\mathcal{H}(T))}=0,
\end{aligned}
$$

where $\mathcal{B}(\mathcal{H}(T))$ is the space of linear bounded operators on $\mathcal{H}(T)$. Thus

$$
\begin{aligned}
w_{\varepsilon}(t, \cdot, y) & =\left(1+i Q_{\varepsilon}\right)^{-1}\left(U_{0}(t-s) \partial_{y} u_{0 \varepsilon}-i Q_{y \varepsilon} u_{\varepsilon}(t, \cdot, y)\right) \\
\longrightarrow \partial_{y} u(t, \cdot, y) & =(1+i Q)^{-1}\left(U_{0}(t) \partial_{y} u_{0}-i Q_{y} u(t, \cdot, y)\right)
\end{aligned}
$$

in $\mathcal{H}(T)$ a.s..

Lemma 3.2. Let $V(\cdot, \cdot, y) \in C^{1}\left(I_{T}, L^{\infty}\left(\mathbb{R}^{n}\right)\right), V_{y}(\cdot, \cdot, y) \in C^{1}\left(I_{T}, L^{\infty}\left(\mathbb{R}^{n}\right)\right)$ and $u_{0}(\cdot, y) \in H^{2}\left(\mathbb{R}^{n}\right)$, $w_{0}(\cdot, y)=\frac{\partial u_{0}}{\partial y}(\cdot, y) \in H^{2}\left(\mathbb{R}^{n}\right)$ for $\rho$-a.e. $y \in \Gamma$. Let $u$ be the solution of (3.2). Then the solution $w(\cdot, \cdot y) \in \mathcal{H}(T)$ of

$$
\begin{gathered}
w(t)=U_{0}(t-s) w_{0}-i \int_{s}^{t} U_{0}(t-\tau) V(\tau) w(\tau) d \tau \\
-i \int_{s}^{t} U_{0}(t-\tau) V_{y}(\tau) u(\tau) d \tau
\end{gathered}
$$

belongs to $\mathcal{Y}(T)$ and it satisfies

$$
i \dot{w}(t, y)=H(t, y) w(t, y)+V_{y}(t, y) u(t, y), \quad H(t, y)=H_{0}+V(t, y) .
$$

Proof. It still suffices to show for $T>0$ small for each $y \in \Gamma$.

For $\rho$-a.e. $y \in \Gamma$, let $v_{h}(t, y)=(w(t+h, y)-w(t, y)) / h$, with $h \neq 0$ small and $|t|<T$. Then $v_{h}(t, y)$ satisfies

$$
v_{h}(t, y)=f_{h}(t, y)-i\left(Q v_{h}\right)(t, y),
$$

where

$$
\begin{aligned}
f_{h}(t, y)=( & \left.U_{0}(t+h)-U_{0}(t)\right) w_{0} / h-i h^{-1} U_{0}(t+h) \int_{0}^{h} U_{0}(-\tau)(V w)(\tau, y) d \tau \\
& -i \int_{0}^{t} U_{0}(t-\tau)\{(V(\tau+h, y)-V(\tau, y)) / h\} w(\tau+h, y) d \tau \\
& -i \int_{0}^{t} U_{0}(t-\tau) V_{y}(\tau, y)\{(u(\tau+h, y)-u(\tau, y)) / h\} d \tau \\
& -i h^{-1} U_{0}(t+h) \int_{0}^{h} U_{0}(-\tau)\left(V_{y} u\right)(\tau, y) d \tau \\
& -i \int_{0}^{t} U_{0}(t-\tau)\left\{\left(V_{y}(\tau+h, y)-V_{y}(\tau, y)\right) / h\right\} u(\tau+h, y) d \tau .
\end{aligned}
$$


Lemma 2.5 tells us that $u \in \mathcal{Y}(T)$ a.s.. Moreover, since $u_{0} \in H^{2}\left(\mathbb{R}^{n}\right), \frac{\partial u_{0}}{\partial y} \in H^{2}\left(\mathbb{R}^{n}\right)$ and $w \in \mathcal{H}(T)$ a.s., it can be seen by Lemma 2.1 that

$$
\begin{aligned}
f_{h}(t, y) \rightarrow f(t, y)=- & i\left[U_{0}(t) V_{y} u(0, y)+S V_{y} \dot{u}(t, y)+S \dot{V}_{y} u(t, y)\right. \\
& \left.+U_{0}(t)\left(H_{0}+V(0)\right) w_{0}(y)+S V \dot{V} w(t, y)\right] \text { in } \mathcal{H}_{l o c}(T) .
\end{aligned}
$$

Then we have

$$
v_{h}(t, y)=(1+i Q)^{-1} f_{h}(t, y) \rightarrow(1+i Q)^{-1} f(t, y) \text { in } \mathcal{H}_{l o c}(T) .
$$

This proves that $\dot{w}(t, y) \in \mathcal{H}(T)$ and

$$
\begin{aligned}
i \dot{w}(t, y)= & U_{0}(t) V_{y} u(0, y)+S V_{y} \dot{u}(t, y)+S \dot{V}_{y} u(t, y) \\
& +U_{0}(t)\left(H_{0}+V(0)\right) w_{0}(y)+S \dot{V} w(t, y)+S V \dot{w}(t, y) .
\end{aligned}
$$

Thus $V w \in \mathcal{Y}^{*}(T)$ a.s.. Lemmas 2.1 and 2.4 imply that $w \in \mathcal{Y}(T)$ with $u \in \mathcal{Y}(T), u_{0} \in H^{2}\left(\mathbb{R}^{n}\right)$ and $w_{0} \in H^{2}\left(\mathbb{R}^{n}\right)$ a.s.. And Eq. (2.14) indicates (3.19).

Now we are ready to present the following theorem on stochastic regularity:

Theorem 3.4. Assume that for $\rho$-a.e. $y \in \Gamma$,

- $V(\cdot, \cdot, y) \in \mathcal{M}(p, \alpha, \beta, T)$,

- $\frac{\partial V}{\partial y} \in L_{\rho}^{2}\left(\Gamma, L^{2}\left(I_{T}, L^{\infty}\left(\mathbb{R}^{n}\right)\right)\right)$,

- $u_{0} \in L_{\rho}^{\infty}\left(\Gamma, L^{2}\left(\mathbb{R}^{n}\right)\right)$,

- $\frac{\partial u_{0}}{\partial y} \in L_{\rho}^{2}\left(\Gamma, L^{2}\left(\mathbb{R}^{n}\right)\right)$

then the integral equation (3.2) admits a unique solution $u \in H_{\rho}^{1}\left(\Gamma, L^{\infty}\left(I_{T}, L^{2}\left(\mathbb{R}^{n}\right)\right)\right)$.

Proof. It's sufficient to consider the well-posedness of (3.5).

Since $\frac{\partial V}{\partial y} \in L_{\rho}^{2}\left(\Gamma, L^{2}\left(I_{T}, L^{\infty}\left(\mathbb{R}^{n}\right)\right)\right)$, we know that $\frac{\partial V}{\partial y} \in L^{2}\left(I_{T}, L^{\infty}\left(\mathbb{R}^{n}\right)\right)$ a.s. and we can choose $\tilde{\beta}=\min (\beta, 2)$ such that $V(\cdot, \cdot, y), \frac{\partial V}{\partial y} \in \mathcal{M}(p, \alpha, \tilde{\beta}, T)$ for $\rho$-a.e. $y \in \Gamma$. We still denote $\tilde{\beta}$ as $\beta$. By the above discussion, it's easy to see that (3.5) admits a unique solution $w(\cdot, \cdot, y) \in$ $C\left(I_{T}, L^{2}\left(\mathbb{R}^{n}\right)\right)$ for $\rho$-a.e. $y \in \Gamma$ and we let $w_{0}=\partial_{y} u_{0}$.

To show that $u$ is $H^{1}$ w.r.t. $y$, we first approximate $V(\cdot, \cdot, y)=V_{1}+V_{2} \in L^{p, \alpha}+L^{\infty, \beta}$ by $V_{\varepsilon}$ and $\frac{\partial V}{\partial y}, u_{0}$ by $V_{y \varepsilon}, u_{0 \varepsilon}$. We take $\phi(x) \in C_{0}^{\infty}\left(\mathbb{R}^{n}\right)$ and $\chi(t) \in C_{0}^{\infty}(\mathbb{R})$ such that

$$
\phi(x), \chi(t) \geq 0 \text { and } \int \phi(x) d x=\int \chi(t) d t=1,
$$


and define

$$
\begin{aligned}
& V_{\varepsilon}(t, x, y)=\int V_{1}(t+\varepsilon s, x+\varepsilon z, y) \chi(s) \phi(z) d s d z+\int V_{2}(t+\varepsilon s, x, y) \chi(s) d s, \\
& V_{y \varepsilon}(t, x, y)=\int \frac{\partial V}{\partial y}(t+\varepsilon s, x, y) \chi(s) d s, \\
& u_{0 \varepsilon}(x, y)=\int u_{0}(x+\varepsilon z, y) \phi(z) d z,
\end{aligned}
$$

where we extended $V_{j}(t, x, y), \frac{\partial V}{\partial y}(t, x, y)$ outside $I_{T} \times \mathbb{R}^{n} \times \Omega$ as $V_{j}(t, x, y) \equiv 0, j=1,2$, $\frac{\partial V}{\partial y}(t, x, y) \equiv 0$. Then

$$
\begin{array}{ll}
V_{\varepsilon}(\cdot, \cdot, y) \in C^{1}\left(I_{T}, L^{\infty}\left(\mathbb{R}^{n}\right)\right), & \lim _{\varepsilon \rightarrow 0}\left\|V_{\varepsilon}(\cdot, \cdot, y)-V(\cdot, \cdot, y)\right\|_{\mathcal{M}}=0, \\
V_{y \varepsilon}(\cdot, \cdot, y) \in C^{1}\left(I_{T}, L^{\infty}\left(\mathbb{R}^{n}\right)\right), & \lim _{\varepsilon \rightarrow 0}\left\|V_{y \varepsilon}(\cdot, \cdot, y)-\frac{\partial V}{\partial y}(\cdot, \cdot, y)\right\|_{\mathcal{M}}=0, \\
V_{y \varepsilon}(\cdot, \cdot, y) \in L^{2}\left(I_{T}, L^{\infty}\left(\mathbb{R}^{n}\right)\right), & \lim _{\varepsilon \rightarrow 0}\left\|V_{y \varepsilon}(\cdot, \cdot, y)-\frac{\partial V}{\partial y}(\cdot, \cdot, y)\right\|_{\infty, 2}=0, \\
u_{0 \varepsilon}(\cdot, y) \in H^{2}\left(\mathbb{R}^{n}\right), & \lim _{\varepsilon \rightarrow 0}\left\|u_{0 \varepsilon}(\cdot, y)-u_{0}(\cdot, y)\right\|_{2}=0, \\
w_{0 \varepsilon}(\cdot, y)=\frac{\partial u_{0 \varepsilon}}{\partial y}(\cdot, y) \in H^{2}\left(\mathbb{R}^{n}\right), & \lim _{\varepsilon \rightarrow 0}\left\|\frac{\partial u_{0 \varepsilon}}{\partial y}(\cdot, y)-\frac{\partial u_{0}}{\partial y}(\cdot, y)\right\|_{2}=0 .
\end{array}
$$

Then Lemma 2.3, 3.1 and 3.2 imply that the solution $u_{\varepsilon}$ of (3.13) and $w_{\varepsilon} \in \mathcal{Y}(T)$ of (3.14) satisfy

$$
\begin{aligned}
& \left\|u_{\mathcal{\varepsilon}}(\cdot, \cdot, y)-u(\cdot, \cdot, y)\right\|_{\mathcal{H}(T)} \rightarrow 0, \\
& i \frac{d}{d t} w_{\varepsilon}=\left(H_{0}+V_{\varepsilon}(t, y)\right) w_{\varepsilon}+V_{y \varepsilon} u_{\varepsilon}(t, y), \\
& \left\|w_{\mathcal{E}}(\cdot, \cdot, y)-w(\cdot, \cdot, y)\right\|_{\mathcal{H}(T)} \rightarrow 0 .
\end{aligned}
$$

Since $H_{0}+V_{\varepsilon}(t, y)$ is self-adjoint in the space $H^{2}\left(\mathbb{R}^{n}\right)$, we see from (3.28b) and Young's inequality that

$$
\frac{d}{d t}\left\|w_{\varepsilon}(t, y)\right\|_{2}^{2} \leq C\left\|w_{\varepsilon}(t, y)\right\|_{2}^{2}+C\left\|V_{y \varepsilon} u_{\varepsilon}(t, y)\right\|_{2}^{2} .
$$

Note that in the proof of Theorem 3.1, we have $u \in L_{\rho}^{\infty}\left(\Gamma, L^{\infty}\left(I_{T}, L^{2}\left(\mathbb{R}^{n}\right)\right)\right.$. Using Gronwall's inequality and let $\varepsilon \rightarrow 0$, we have

$$
\|w(t)\|_{2}^{2} \leq C(T)\left(\left\|w_{0}\right\|_{2}^{2}+\left\|\frac{\partial V}{\partial y}\right\|_{\infty, 2}^{2}\|u\|_{2, \infty}^{2}\right), \quad \forall t \in I_{T}
$$

where $C(T)$ is a constant dependent on $T$. Then taking the supremum in $t$ over $I_{T}$ and 
integrating w.r.t. $\rho(y) d y$. We have

$$
\begin{aligned}
& \|w\|_{L_{\rho}^{2}\left(\Gamma, L^{\infty}\left(I_{T}, L^{2}\left(\mathbb{R}^{n}\right)\right)\right)}^{2} \\
\leq & C(T)\left(\left\|w_{0}\right\|_{L^{2}\left(\mathbb{R}^{n}\right) \otimes L_{\rho}^{2}(\Gamma)}^{2}+\left\|\frac{\partial V}{\partial y}\right\|_{L_{\rho}^{2}\left(\Gamma, L^{2}\left(I_{T}, L^{\infty}\left(\mathbb{R}^{n}\right)\right)\right)}^{2}\|u\|_{L_{\rho}^{\infty}\left(\Gamma, L^{\infty}\left(I_{T}, L^{2}\left(\mathbb{R}^{n}\right)\right)\right)}^{2}\right) .
\end{aligned}
$$

Hence $u \in H_{\rho}^{1}\left(\Gamma, L^{\infty}\left(I_{T}, L^{2}\left(\mathbb{R}^{n}\right)\right)\right)$.

Theorem 3.5. Assume that for $\rho$-a.e. $y \in \Gamma$,

- $V(\cdot, \cdot, y) \in \mathcal{M}(p, \alpha, \beta, T)$,

- $\frac{\partial^{(j)} V}{\partial y^{(j)}} \in L_{\rho}^{\infty}\left(\Gamma, L^{2}\left(I_{T}, L^{\infty}\left(\mathbb{R}^{n}\right)\right)\right), j=1, \cdots, k-1$,

- $\frac{\partial^{(k)} V}{\partial y^{(k)}} \in L_{\rho}^{2}\left(\Gamma, L^{2}\left(I_{T}, L^{\infty}\left(\mathbb{R}^{n}\right)\right)\right)$,

- $u_{0} \in L_{\rho}^{\infty}\left(\Gamma, L^{2}\left(\mathbb{R}^{n}\right)\right)$,

- $\frac{\partial^{(j)} u_{0}}{\partial y^{(j)}} \in L_{\rho}^{2}\left(\Gamma, L^{2}\left(\mathbb{R}^{n}\right)\right), \forall j=1, \cdots, k$,

Then (3.2) has a unique solution $u \in H_{\rho}^{k}\left(\Gamma, L^{\infty}\left(I_{T}, L^{2}\left(\mathbb{R}^{n}\right)\right)\right)$.

The proof can be done by induction.

Remark 3.3. Similarly, in Theorem 3.5, an upper bound $C(T, k)$ on the $H^{k}$-norm of $u$ would be given. It is also dependent on $k$ and T. It could blow up as $k \rightarrow \infty$. And it may also blow up as $T \rightarrow \infty$.

Remark 3.4. The above stochastic regularity analysis is performed for the linear Schrödinger equation. To consider such an analysis for the Schrödinger equation with a nonlinear potential, well-posedness results on the nonlinear Schrödinger equation in the deterministic case are needed, e.g., $[10,11]$, etc. The techniques can be totally different and much harder. And stricter conditions may be needed to ensure the regularity. But it is a topic worthwhile for subsequent researches.

\section{Time splitting based stochastic collocation method and convergence results}

As a usual practice of numerical computations, we compute the Schrödinger equation on a truncated bounded domain. For simplicity, we consider the 1-dimensional Schrödinger 
equation on an interval $[a, b]$ with periodic boundary conditions:

$$
\left\{\begin{array}{l}
i \frac{\partial u}{\partial t}=-\frac{\partial^{2} u}{\partial x^{2}}+V(x, y) u, \quad t>0, \quad x \in[a, b], \quad y \in \Gamma \\
\left.u\right|_{t=0}=u_{0}(x, y) \\
u(t, a, y)=u(t, b, y) \\
\frac{\partial u}{\partial x}(t, a, y)=\frac{\partial u}{\partial x}(t, b, y),
\end{array}\right.
$$

where $y$ is still a random variable with a density function $\rho(y)$ and $y \in \Gamma \subset \mathbb{R}$.

\subsection{Time splitting based stochastic collocation method}

We combine the time splitting method [8] and the stochastic collocation method.

Given the density function $\rho(y)$ of $y$, we can find its corresponding orthogonal polynomial. Assume that $\left\{y_{n}\right\}_{n=0}^{N}$ are the roots of orthogonal polynomial of order $N+1$. For each $y_{n}$, using the time splitting method, we solve

$$
\left\{\begin{array}{l}
i \frac{\partial u}{\partial t}=-\frac{\partial^{2} u}{\partial x^{2}}+V\left(x, y_{n}\right) u, \quad t>0, \quad x \in[a, b] \\
\left.u\right|_{t=0}=u_{0}\left(x, y_{n}\right) \\
u(t, a, y)=u(t, b, y) \\
\frac{\partial u}{\partial x}(t, a, y)=\frac{\partial u}{\partial x}(t, b, y) .
\end{array}\right.
$$

We choose spatial step $\Delta x=(b-a) / M$, where $M$ is an even number and time step $\Delta t>0$. And we let $x_{j}=a+j \Delta x, t_{m}=m \Delta t$ and $U_{j}^{m}\left(y_{n}\right)$ be the approximation of $u\left(t_{m}, x_{j}, y_{n}\right)$.

From $t_{m}$ to $t_{m+1}$, we solve (4.2) in the following two steps:

$$
\begin{aligned}
& U_{j}^{m+1 / 2}\left(y_{n}\right)=\frac{1}{M} \sum_{\ell=M / 2}^{M / 2-1} e^{-i \Delta t \mu_{\ell}^{2} / 2} \hat{U}_{\ell}^{m}\left(y_{n}\right) e^{i \mu_{\ell}\left(x_{j}-a\right)}, \quad j=0,1, \cdots, M-1, \\
& U_{j}^{m+1}\left(y_{n}\right)=e^{-i V\left(x_{j}, y_{n}\right) \Delta t} U_{j}^{m+1 / 2}\left(y_{n}\right),
\end{aligned}
$$

where $\mu_{\ell}=2 \pi \ell /(b-a)$,

$$
\begin{array}{ll}
\hat{U}_{\ell}^{m}\left(y_{n}\right)=\sum_{j=0}^{M-1} U_{j}^{m}\left(y_{n}\right) e^{-i \mu_{\ell}\left(x_{j}-a\right)}, & \ell=-\frac{M}{2}, \cdots, \frac{M}{2}-1, \\
U_{j}^{0}\left(y_{n}\right)=u_{0}\left(x_{j}, y_{n}\right), & j=0,1, \cdots, M .
\end{array}
$$

Let $u_{I}^{m}\left(x, y_{n}\right)$ be the trigonometric interpolation using $\left\{\left(x_{j}, U_{j}^{m}\left(y_{n}\right)\right)\right\}_{j=0}^{M}$

$$
u_{I}^{m}\left(x, y_{n}\right)=\frac{1}{M} \sum_{\ell=-M / 2}^{M / 2} \hat{U}_{\ell}^{m}\left(y_{n}\right) e^{i \mu_{\ell}(x-a)},
$$


where $\hat{U}_{\ell}^{m}\left(y_{n}\right)$ is defined as above. And let $I_{N} u_{I}^{m}(x, y)$ be the Lagrange interpolation using $\left\{\left(y_{n}, u_{I}^{m}\left(x, y_{n}\right)\right)\right\}_{n=0}^{N}$

$$
I_{N} u_{I}^{m}(x, y)=\sum_{n=0}^{N} u_{I}^{m}\left(x, y_{n}\right) p_{n}(y)
$$

where

$$
p_{n}(y)=\frac{\left(y-y_{0}\right) \cdots\left(y-y_{n-1}\right)\left(y-y_{n+1}\right) \cdots\left(y-y_{N}\right)}{\left(y_{n}-y_{0}\right) \cdots\left(y_{n}-y_{n-1}\right)\left(y_{n}-y_{n+1}\right) \cdots\left(y_{n}-y_{N}\right)} .
$$

Then $I_{N} u_{I}^{m}(x, y)$ is our numerical approximation.

\subsection{Convergence analysis}

Since the convergence of the stochastic collocation method is closely related to the regularity of the solution of Eq. (4.1), $u(x, y)$, w.r.t. $y$, we still need to first analyse the regularity of $u(x, y)$ w.r.t. $y$. We assume the derivative of $u$ w.r.t. $y$ exists. We give sufficient conditions to ensure the integrability of the derivative of $u$ w.r.t. $\rho(y) d y$.

Theorem 4.1. Assume there exists a constant $C>0$ such that

- For $\rho$-a.e. $y \in \Gamma, \max _{a \leq x \leq b} \partial_{y}^{k}|V(x, y)| \leq C, k=1, \cdots, K-1$,

- $\int_{\Gamma} \max _{a \leq x \leq b}\left|\partial_{y}^{K} V(x, y)\right|^{2} \rho(y) d y \leq C$,

- For $\rho$-a.e. $y \in \Gamma, \int_{a}^{b}\left|u_{0}(x, y)\right|^{2} d x \leq C$,

- $\int_{\Gamma} \int_{a}^{b}\left|\partial_{y}^{k} u_{0}(x, y)\right|^{2} d x \rho(y) d y \leq C, k=1, \cdots, K$.

We have $\partial_{y}^{K} u(T, x, y) \in L_{\rho}^{2}(\Gamma)$, a.e. $x \in[a, b]$ and

$$
\int_{\Gamma} \int_{a}^{b}\left|\partial_{y}^{K} u(T, x, y)\right|^{2} \rho(y) d x d y \leq C(T, K) .
$$

Proof. First we have

$$
\bar{u} \partial_{t} u=\frac{i}{2} \bar{u} \partial_{x}^{2} u-i \bar{u} V u .
$$

Taking the conjugate of (4.9) and adding it with (4.9), we integrate it w.r.t $x$ on $[a, b]$. And we have

$$
\partial_{t} \int_{a}^{b}|u(t, x, y)|^{2} d x=0
$$

By the assumption on the initial data, we have

$$
\int_{a}^{b}|u(t, x, y)|^{2} d x=\int_{a}^{b}\left|u_{0}(x, y)\right|^{2} d x \leq C, \quad \rho \text {-a.e. } y \in \Gamma, \quad 0 \leq t \leq T,
$$


i.e., $u(t, \cdot, \cdot) \in L^{\infty}\left(\Gamma, L^{2}([a, b])\right), 0 \leq t \leq T$. We proceed to show that

$$
\int_{a}^{b}\left|\partial_{y}^{k} u(t, x, y)\right|^{2} d x \leq C(T, k) f_{k}(y), \quad k=0,1, \cdots, K-1, \quad 0 \leq t \leq T,
$$

where $f_{k}(y)$ is integrable w.r.t. $\rho(y) d y$. We proceed by induction on $k$. We have proved the case where $k=0$. Assume that it holds for $\ell \leq k-1$. For $k$, we have $\partial_{y}^{k} u$ satisfying

$$
i \partial_{t} \partial_{y}^{k} u=-\frac{1}{2} \partial_{x}^{2} \partial_{y}^{k} u+V \partial_{y}^{k} u+\sum_{\ell=1}^{k}\left(\begin{array}{l}
k \\
\ell
\end{array}\right) \partial_{y}^{\ell} V \partial_{y}^{k-\ell} u .
$$

Repeating the above deduction, we have

$$
\partial_{t} \int_{a}^{b}\left|\partial_{y}^{k} u(t, x, y)\right|^{2} d x=-\int_{a}^{b} 2 \operatorname{Re}\left(i \partial_{y}^{k} \bar{u} \sum_{\ell=1}^{k}\left(\begin{array}{l}
k \\
\ell
\end{array}\right) \partial_{y}^{\ell} V \partial_{y}^{k-\ell} u\right) d x .
$$

By Young's inequality and Hölder's inequality, we have

$$
\begin{aligned}
\partial_{t} \int_{a}^{b}\left|\partial_{y}^{k} u(t, x, y)\right|^{2} d x & \leq \int_{a}^{b}\left|\partial_{y}^{k} u(t, x, y)\right|^{2} d x+\int_{a}^{b}\left|\sum_{\ell=1}^{k}\left(\begin{array}{l}
k \\
\ell
\end{array}\right) \partial_{y}^{\ell} V \partial_{y}^{k-\ell} u\right|^{2} d x \\
& \leq \int_{a}^{b}\left|\partial_{y}^{k} u(t, x, y)\right|^{2} d x+C(k) \sum_{\ell=1}^{k} \max _{x \in[a, b]} \partial_{y}^{\ell} V^{2} \int_{a}^{b}\left|\partial_{y}^{k-\ell} u\right|^{2} d x
\end{aligned}
$$

By Gronwall's inequality, we have

$$
\begin{aligned}
& \int_{a}^{b}\left|\partial_{y}^{k} u(t, x, y)\right|^{2} d x \\
\leq & C(T, k)\left(\int_{a}^{b}\left|\partial_{y}^{k} u_{0}\right|^{2} d x+\sum_{\ell=1}^{k} \max _{x \in[a, b]} \partial_{y}^{\ell} V^{2} \int_{0}^{T} \int_{a}^{b}\left|\partial_{y}^{k-\ell} u(s, x, y)\right|^{2} d x d s\right) .
\end{aligned}
$$

By induction, we have

$$
\int_{a}^{b}\left|\partial_{y}^{k} u(t, x, y)\right|^{2} d x \leq C(T, k)\left(\int_{a}^{b}\left|\partial_{y}^{k} u_{0}\right|^{2} d x+\sum_{\ell=1}^{k} \max _{x \in[a, b]} \partial_{y}^{\ell} V^{2} f_{k-\ell}(y)\right) .
$$

And by the assumptions on $\partial_{y}^{k} u_{0}$ and $\partial_{y}^{\ell} V, \ell=1, \cdots, k$, we have

$$
\int_{a}^{b}\left|\partial_{y}^{k} u(t, x, y)\right|^{2} d x \leq C(T, k) f_{k}(y), \quad 0 \leq t \leq T,
$$

where $f_{k}(y)$ is integrable w.r.t. $\rho(y) d y$. For $\partial_{y}^{K} u$, repeating the above deduction, we have

$$
\begin{aligned}
& \int_{a}^{b}\left|\partial_{y}^{K} u(t, x, y)\right|^{2} d x \\
\leq & C(T, K)\left(\int_{a}^{b}\left|\partial_{y}^{K} u_{0}\right|^{2} d x+\sum_{k=1}^{K-1} f_{k}(y)+\max _{x \in[a, b]} \partial_{y}^{K} V^{2} \int_{0}^{T} \int_{a}^{b}|u(t, x, y)|^{2} d x d t\right) .
\end{aligned}
$$


Note that $\int_{a}^{b}|u(t, x, y)|^{2} d x \leq C$ for $\rho$-a.e. $y \in \Gamma$. Integrating both sides of the above inequality w.r.t. $\rho(y) d y$, we have

$$
\int_{\Gamma} \int_{a}^{b}\left|\partial_{y}^{K} u(t, x, y)\right|^{2} \rho(y) d x d y \leq C(T, K)+C(T, K) \int_{\Gamma x \in[a, b]} \max _{y}^{K} V^{2} \rho(y) d y .
$$

By our assumptions on $\partial_{y}^{K} V$, we have

$$
\int_{\Gamma} \int_{a}^{b}\left|\partial_{y}^{K} u(t, x, y)\right|^{2} \rho(y) d x d y \leq C(T, K)
$$

In particular,

$$
\int_{\Gamma} \int_{a}^{b}\left|\partial_{y}^{K} u(T, x, y)\right|^{2} \rho(y) d x d y \leq C(T, K)
$$

Moreover, by Tonelli theorem, $\partial_{y}^{K} u(T, x, y) \in L_{\rho}^{2}(\Gamma)$ for a.e. $x \in[a, b]$.

Remark 4.1. In Theorem 4.1, the constant $C(T, K)$ may blow up as $k \rightarrow \infty$. Also, this constant $C(T, K)$ could blow up as $T \rightarrow \infty$.

We then consider the convergence analysis. We define the mean error

$$
e_{\text {mean }}\left(t_{m}\right)=E\left[\left|u\left(t_{m}\right)-I_{N} u_{I}^{m}\right|\right],
$$

and the mean square error

$$
e_{m s}\left(t_{m}\right)=M\left[\left|u\left(t_{m}\right)-I_{N} u_{I}^{m}\right|\right]
$$

where

$$
\begin{aligned}
& E[f]=\int_{a}^{b} \int_{\Gamma} f(x, y) \rho(y) d y d x, \\
& M[f]=\left(\int_{a}^{b} \int_{\Gamma} f(x, y)^{2} \rho(y) d y d x\right)^{1 / 2} .
\end{aligned}
$$

Lemma 4.1. Assume a given function $f(y)$ satisfies $f \in H^{k}(-1,1)$ and let $I_{N} f$ be its interpolation polynomial associated with the $N+1$ Gauss, Gauss-Radau or Gauss-Lobatto points. Then for $k \leq N$ the following estimate holds

$$
\left\|f-I_{N} f\right\|_{L^{2}(-1,1)} \leq C N^{-k}\left\|f^{(k)}\right\|_{L^{2}(-1,1)} .
$$

Remark 4.2. The above lemma is an estimate for the Gauss-Legendre interpolation error whose proof can be found in Section 5.6 of [9]. Estimates for other common density functions $\rho$ are also presented in [9], such as the normal distribution, exponential distribution, etc. 
By the above interpolation theorem, we have

Theorem 4.2. Assume $y$ is a random variable uniformly distributed on $[-1,1]$. Under the assumption of 4.1 , we have

$$
\int_{a}^{b} \int_{\Gamma}\left(u(T)-I_{N} u(T)\right)^{2} \rho(y) d y d x \leq \frac{C(T, K)}{N^{2 K}} .
$$

Proof. By Theorem 4.1, $\partial_{y}^{K} u(T, x, y) \in L_{\rho}^{2}(\Gamma)$ for a.e. $x \in[a, b]$. Hence by Lemma 4.1,

$$
\left(\int_{\Gamma}\left(u(T)-I_{N} u(T)\right)^{2} \rho(y) d y\right) \leq C N^{-2 K}\left(\int_{\Gamma}\left|\partial_{y}^{K} u\right|^{2} \rho(y) d y\right) .
$$

Integrating both sides w.r.t. $x$, we have

$$
\int_{a}^{b} \int_{\Gamma}\left(u(T)-I_{N} u(T)\right)^{2} \rho(y) d y d x \leq C N^{-2 K} \int_{\Gamma} \int_{a}^{b}\left|\partial_{y}^{K} u(T, x, y)\right|^{2} \rho(y) d x d y \leq \frac{C(T, K)}{N^{2 K}} .
$$

Thus, we complete the proof.

For the time splitting method, from [8], we have

Theorem 4.3. Assume for $\rho$-a.e. $y \in \Gamma$,

- $V(x, y)$ is $C^{\infty}$ in $x$ and $(b-a)$-periodic,

- there exists constant $E_{L_{1}+L_{2}}(T)>0$ irrelevant to $x, y$ such that

$$
\left\|\frac{\partial^{L_{1}+L_{2}}}{\partial x^{L_{1}} \partial_{t}^{L_{2}}} u\right\|_{C\left([0, T] ; L^{2}(a, b)\right)} \leq E_{L_{1}+L_{2}}(T),
$$

- there exists constant $F_{L}>0$ irrelevant to $x, y$ such that

$$
\left\|\partial_{x}^{L} V\right\|_{L^{\infty}(a, b)} \leq F_{L} .
$$

Then we have

$$
\left\|u\left(t_{m}, \cdot, y\right)-u_{I}^{m}(\cdot, y)\right\|_{2} \leq C(T, L) \frac{T}{\Delta t} \Delta x^{L}+D(T) T \Delta t, \quad 0 \leq t_{m} \leq T, \quad \rho-\text { a.e. } y \in \Gamma .
$$

Remark 4.3. In Theorem 4.3, the constant $C(T, L)$ in the final error estimate is related to the regularity of $u$ w.r.t. $t$ and $x$ and the regularity of $V$ w.r.t. $x$ in the domain $[0, T] \times[a, b]$ up to the order $L$ and hence it can be dependent on $T$ and $L$. Similarly, the constant $D(T)$ is related to the regularity of $u$ w.r.t. $t$ and $x$ and the regularity of $V$ w.r.t. $x$ in the domain $[0, T] \times[a, b]$ up to the order 2 and so it can be dependent on $T$.

Combining the above two theorem, we have 
Theorem 4.4. Under the assumptions of Theorems 4.2 and 4.3 , we have

$$
\begin{array}{ll}
e_{\text {mean }}\left(t_{m}\right) \leq \frac{C(T, K)}{N^{K}}+C(T, L) \frac{T}{\Delta t} \Delta x^{L}+D(T) T \Delta t, & 0 \leq t_{m} \leq T, \\
e_{m s}\left(t_{m}\right) \leq \frac{C(T, K)}{N^{K}}+C(T, L) \frac{T}{\Delta t} \Delta x^{L}+D(T) T \Delta t, & 0 \leq t_{m} \leq T .
\end{array}
$$

Proof. For the means square error,

$$
e_{m s}\left(t_{m}\right) \leq M\left[u-I_{N} u\right]\left(t_{m}\right)+M\left[I_{N} u\left(t_{m}\right)-I_{N} u_{I}^{m}\right]
$$

By Theorem of 4.2, we have

$$
M\left[u-I_{N} u\right]\left(t_{m}\right) \leq\left(\int_{a}^{b} C N^{-2 K}\left|u\left(t_{m}\right)\right|_{H_{\rho}^{K}(\Gamma)}^{2} d x\right)^{1 / 2}=C(T, K) N^{-K} .
$$

By the orthonormality of the interpolation basis polynomial, we have

$$
M\left[I_{N} u\left(t_{m}\right)-I_{N} u_{I}^{m}\right] \leq\left(\sum_{j=0}^{N} A_{j}\left\|u\left(t_{m}, x, y_{j}\right)-u_{I}^{m}\left(x, y_{j}\right)\right\|_{L^{2}([a, b])}^{2}\right)^{1 / 2},
$$

where $A_{j} \geq 0$ is the weight of Gauss quadrature and $\sum_{j=0}^{N} A_{j}=1$. By Theorem 4.3 , we have

$$
\left\|u\left(t_{m}, x, y_{j}\right)-u_{I}^{m}\left(x, y_{j}\right)\right\|_{L^{2}([a, b])} \leq C(T, L) \frac{T}{\Delta t} \Delta x^{L}+D(T) T \Delta t, \quad j=0,1, \cdots, N .
$$

Hence

$$
M\left[I_{N} u\left(t_{m}\right)-I_{N} u_{I}^{m}\right] \leq C(T, L) \frac{T}{\Delta t} \Delta x^{L}+D(T) T \Delta t .
$$

And we obtain the estimate for $e_{m s}$.

For the mean error, by Hölder's inequality

$$
e_{\text {mean }}\left(t_{m}\right)^{2} \leq(b-a) e_{m s}\left(t_{m}\right)^{2}
$$

and we obtain the estimate for it.

We also consider the error estimate of statistical moments. We define the error of the first moment

$$
e_{1 m}\left(t_{m}\right)=\left\|\mathbb{E}\left[u\left(t_{m}, x\right)\right]-\mathbb{E}\left[I_{N} u_{I}^{m}(x)\right]\right\|_{L^{1}([a, b])}
$$

and the error of the second moment

$$
e_{2 m}\left(t_{m}\right)=\left\|\left(\mathbb{E}\left[\left|u\left(t_{m}, x\right)\right|^{2}\right]\right)^{1 / 2}-\left(\mathbb{E}\left[\left|I_{N} u_{I}^{m}(x)\right|^{2}\right]\right)^{1 / 2}\right\|_{L^{2}([a, b])} .
$$


Theorem 4.5. Under the assumption of Theorem 4.4, we have

$$
\begin{array}{ll}
e_{1 m}\left(t_{m}\right) \leq \frac{C(T, K)}{N^{K}}+C(T, L) \frac{T}{\Delta t} \Delta x^{L}+D(T) T \Delta t, & 0 \leq t_{m} \leq T, \\
e_{2 m}\left(t_{m}\right) \leq \frac{C(T, K)}{N^{K}}+C(T, L) \frac{T}{\Delta t} \Delta x^{L}+D(T) T \Delta t, & 0 \leq t_{m} \leq T .
\end{array}
$$

Proof. Just note that

$$
\begin{aligned}
& e_{1 m}\left(t_{m}\right) \leq e_{\text {mean }}\left(t_{m}\right), \\
& e_{2 m}\left(t_{m}\right) \leq e_{m s}\left(t_{m}\right),
\end{aligned}
$$

and we can prove the theorem.

Remark 4.4. In Theorems 4.4 and 4.5 , the constant $C(T, K)$ is related to the regularity of $u$ or $|u|^{2}$ w.r.t. $y$ in the domain $[0, T] \times[a, b]$ up to the order $K$. In Theorem 4.1, we give an upper bound for the $L_{\rho}^{2}(\Gamma,[a, b])$-norm of $\partial_{y}^{K} u(T)$ and the bound $C(T, K)$ increases as $T$ grows according to the proof of Theorem 4.1. However, if the true regularity of $u$ or $|u|^{2}$ w.r.t. $y$ in the domain $[0, T] \times[a, b]$ is independent of $T$, the error originating from the stochastic collocation method will be independent of $T$. Our numerical experiment will show this phenomenon.

As for the constants $C(T, L)$ and $D(T)$, as is stated, they are related to the regularity of $u$ or $|u|^{2}$ w.r.t. $t$ and $x$ and the regularity of $V$ w.r.t. $x$ in the domain $[0, T] \times[a, b]$. Their dependence on $T$ is problem-dependent as well.

Remark 4.5. As in Section 3, the analysis in this section is limited to the linear case. Extension to the nonlinear case is not straightforward. New techniques may be needed and it is much harder. We have not really investigated this problem and it is a topic for our subsequent research.

\section{$5 \quad$ Numerical experiments}

We consider the Schrödinger equation on $[-\pi, \pi]$. And we give 3 numerical examples to support our convergence analysis and 1 numerical example to study the dependence of errors on different final times $T$.

Example 5.1. Consider the potential

$$
V(x, y)=\frac{1}{2} G(y)^{8} x^{2}
$$

and initial data

$$
u_{0}(x, y)=\frac{G(y)}{\pi^{1 / 4}} \exp \left(-\frac{1}{2} G(y)^{4} x^{2}\right)
$$




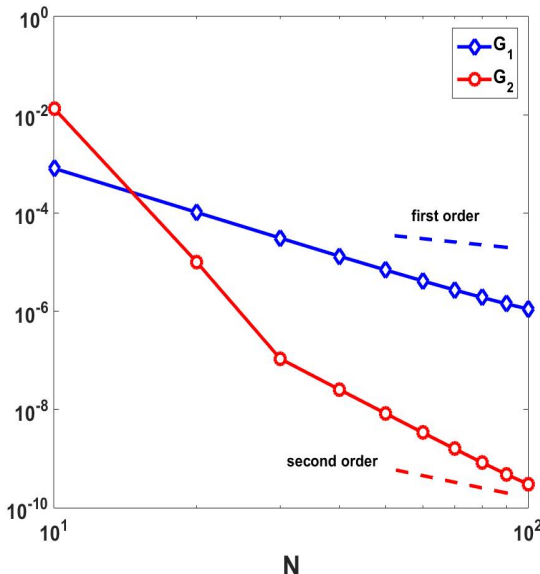

(a) $e_{\text {mean }}$

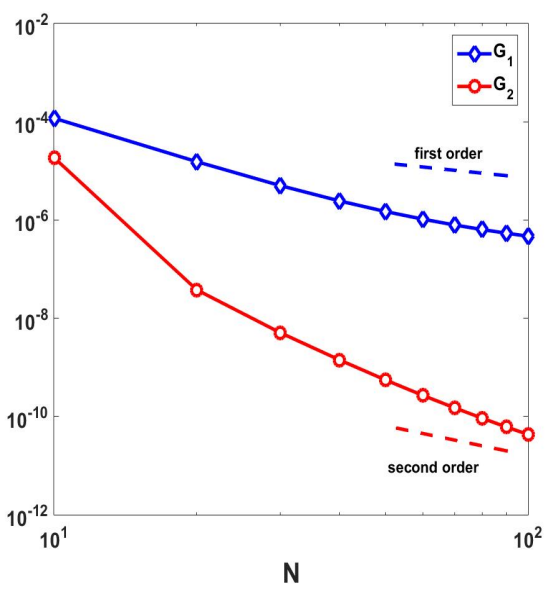

(c) $e_{1 m}$

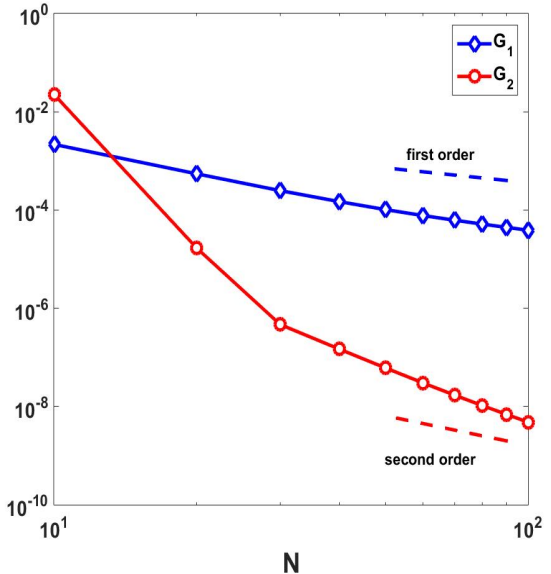

(b) $e_{m s}$

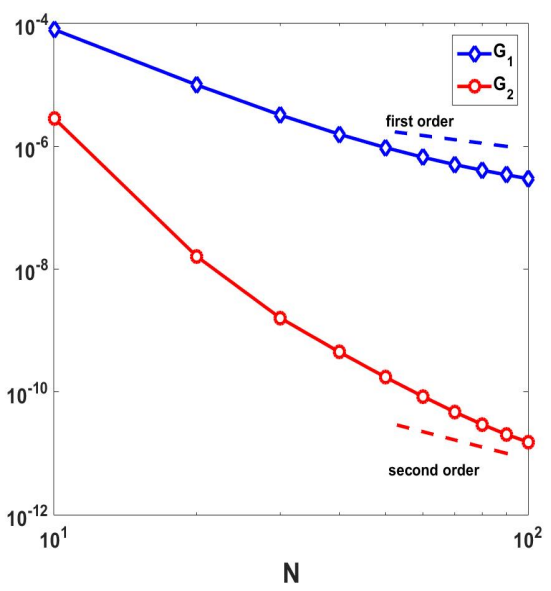

(d) $e_{2 m}$

Figure 1: Convergence of Example 5.1.

where $G(y)$ can be $G_{1}(y)=\frac{1}{2} \exp \left(y^{3 / 5}\right)+1$ and $G_{2}(y)=\frac{1}{2} \exp \left(y^{8 / 5}\right)+1$. And $y$ is a random variable uniformly distributed on $[0,1]$. By Theorems 4.4 and 4.5 , we know that the convergence is at least of order 1 when $G=G_{1}$ and at least of order 2 when $G=G_{2}$. And we can take

$$
u(t, x, y)=\frac{G(y)}{\pi^{1 / 4}} \exp \left(-\frac{1}{2} G(y)^{4} x^{2}\right) \exp \left(-\frac{i}{2} G(y)^{4} t\right)
$$

as the reference solution.

We consider the convergence w.r.t. the number of collocation point $N$ at $T=0.5$. And the step sizes are $\Delta t=5 e-7, \Delta x=\pi / 512$. The convergence is shown in Fig. 1 .

We can see that when $G=G_{1}$, the convergence order of all the 4 types of errors is 


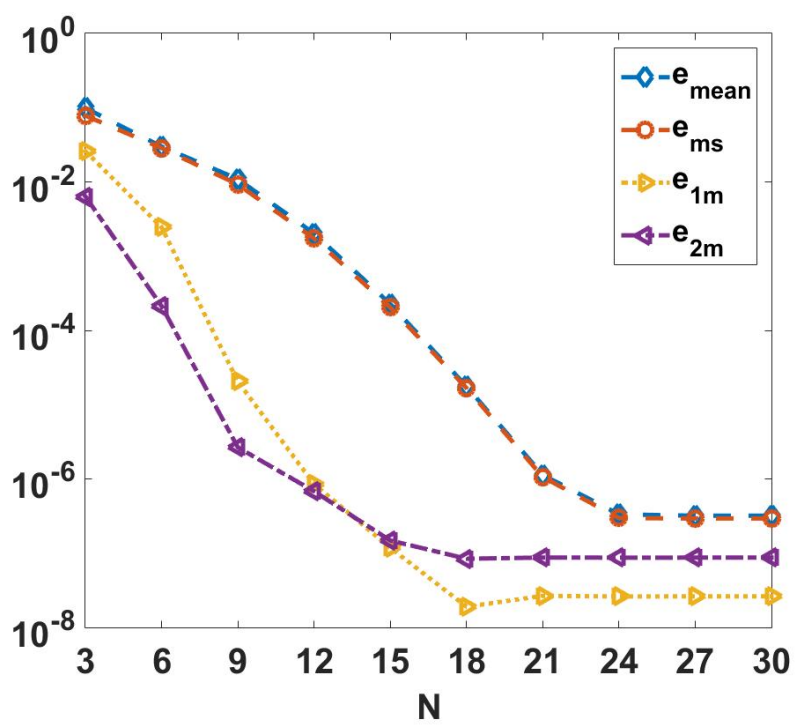

Figure 2: Convergence of Example 5.2.

slightly better than 1 and when $G=G_{2}$, the convergence order is better than 2, which is consistent with our analysis. For $e_{\text {mean }}$, the convergence order is 1 order better than our estimate, which implies our estimate may not be sharp. But we can still see that the more smoothly $V$ and $u_{0}$ are dependent on $y$, the faster is the convergence of the stochastic collocation method.

Example 5.2. Consider the potential

$$
V(x, y)=2 \log (1+y)\left(1-x^{2}\right)^{2}
$$

and the initial data

$$
u_{0}(x, y)=\left(\frac{10}{\pi}\right)^{1 / 4} \exp \left(-5 x^{2}\right)
$$

Here $y$ is still uniformly distributed on $[0,1]$. Since $V$ is $C^{\infty}$ in $y$, we expect spectral convergence.

We consider error at $T=0.5$. And the reference solution is computed using meshes $\Delta t=5 e-7, \Delta x=\pi / 2048$ and collocation point $N=100$. The step sizes for the numerical solution are $\Delta t=1 e-6, \Delta x=\pi / 1024$. The results are shown in Fig. 2.

Example 5.3. Consider the potential

$$
V(x, y)=e^{-y} \cos \left(\frac{1}{2} x\right)
$$




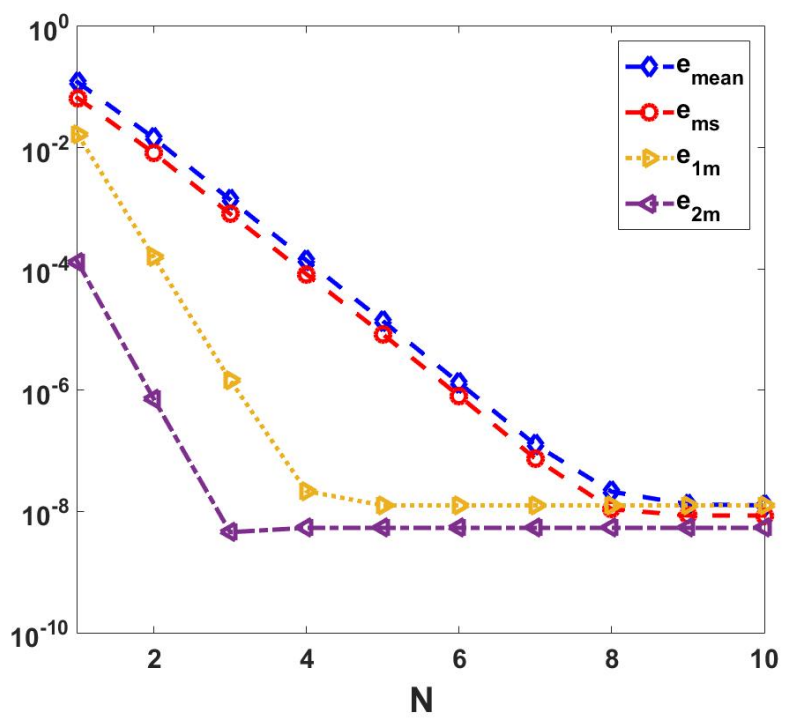

Figure 3: Convergence of Example 5.3.

and initial data

$$
u_{0}(x, y)=\left(\frac{10}{\pi}\right)^{1 / 4} \exp \left(-5 x^{2}\right)
$$

Here $y$ is subject to beta distribution $\operatorname{Beta}(2,2)$. Since $V$ is still $C^{\infty}$ in $y$, we still expect spectral convergence.

We consider errors at $T=0.5$. The reference solution is computed using $\Delta t=5 e-7$, $\Delta x=\pi / 2048$ and collocation point $N=100$. And the step sizes for the numerical solution are $\Delta t=1 e-6, \Delta x=\pi / 1024$. The results are shown in Fig. 3.

In both Examples 5.2 and 5.3, we observe spectral convergence. And in Example 5.3, the convergence is even faster since the beta distribution $\operatorname{Bet} a(2,2)$ is more concentrated than the uniform distribution.

Example 5.4. We again consider the harmonic potential and initial data in Example 5.1. And we choose $G(y)=G_{1}(y)$ here. We consider errors at different final times $T=0.1,0.5,1$. And the step sizes are still $\Delta t=5 e-7, \Delta x=\pi / 512$. The convergence of errors w.r.t. the number of collocation points at different final times is shown in Fig. 4.

We can clearly observe the long-time effect for errors $e_{\text {mean }}(T), e_{m s}(T)$ and $e_{1 m}(T)$ in Fig. 4, which means the errors grow as $T$ grows. As for the error $e_{2 m}(T)$, it is almost the same for $T=0.1,0.5,1$. We can see from the reference solution (5.3) that the density $\|u(T, \cdot, y)\|_{2}$ does not depend on $T$ and hence $e_{2 m}(T)$ does not change as $T$ varies. 


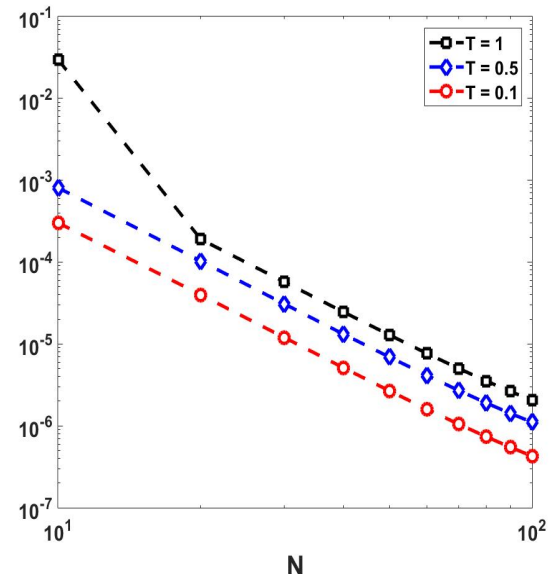

(a) $e_{\text {mean }}$

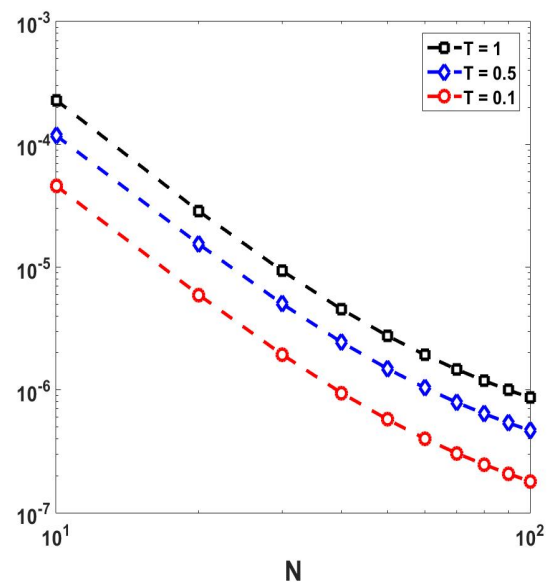

(c) $e_{1 m}$

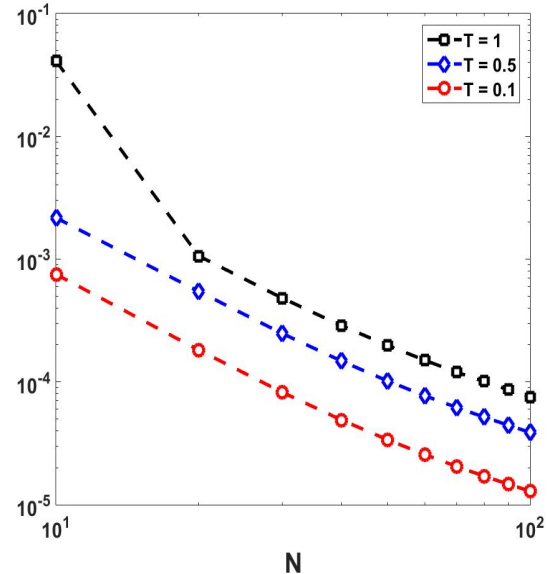

(b) $e_{m s}$

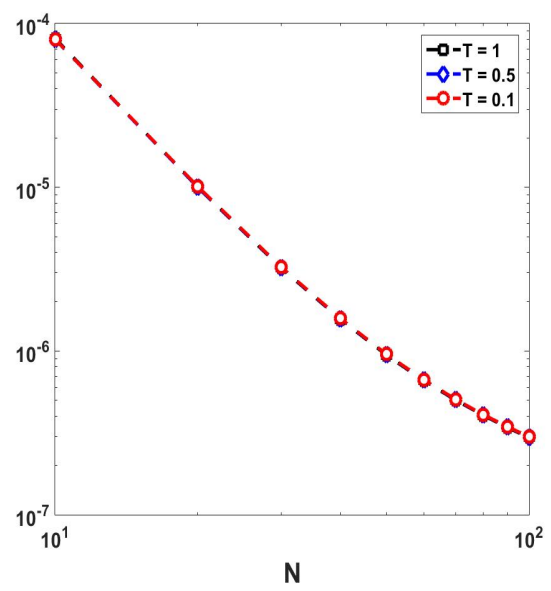

(d) $e_{2 m}$

Figure 4: errors at different final times $T$ in Example 5.4.

\section{Conclusions}

In this work, we present the convergence analysis on the stochastic collocation methods for the Schrödinger equation with random inputs. We analyse the convergence rate w.r.t. the number of collocation points.

We first study the regularity of the solution to the random Schrödinger equation where $x \in \mathbb{R}^{d}$ w.r.t. the random variable. We utilize the well-posedness results in the deterministic case and present two cases of regularity results in the random case. It can be seen that the smoothness of the potential and initial data w.r.t. the random variable is the main factor in determining the stochastic regularity of the solution. And the stochastic 
regularity is also closely associated with the space-time smoothness of the potential and initial data. To accommodate some singularities for the random variables, the space-time regularities should be strengthened.

We then study the random Schrödinger equation on a bounded interval. We present the time splitting based stochastic collocation method. Based on the interpolation theories and convergence results of the time splitting method, we give the convergence analysis of the time splitting based stochastic collocation method and provide sufficient conditions to ensure spectral convergence. Our numerical experiments show that spectral convergence can be observed under appropriate conditions. In conclusion, the stochastic collocation method is a solid method when the magnitude of the randomness is within an appropriate range.

In the future, such analyses can be extended to the nonlinear cases. And to achieve uniform convergence w.r.t. $\varepsilon$ for the random Schrödinger equation in the semiclassical regime, new sampling method should be developed. Moreover, the modeling and algorithm design of highly random physical phenomena should be considered.

\section{Acknowledgements}

This work was partially supported by the National Key Research and Development Plan of China No. 2017YFC0601801 and NSFC Project No. 11871298.

\section{References}

[1] Philip W Anderson, Absence of diffusion in certain random lattices, Phys. Rev., 109(5) (1958), pp. 1492-1505.

[2] Y AVISHAI, RM REDHEFFER AND YB BAND, Electron states in a magnetic field and random impurity potential: use of the theory of entire functions, J. Phys. A Math. Gen. 25(13) (1992), pp. 3883-3889.

[3] IVo BABUŠKa, FABio Nobile And RAúl TEMPONE, A stochastic collocation method for elliptic partial differential equations with random input data, SIAM J. Numer. Anal., 45(3) (2007), pp. 1005-1034.

[4] IVO BABUŠKA, RAÚl TEMPONE AND GEORGIOS E ZOURARIS, Galerkin finite element approximations of stochastic elliptic partial differential equations, SIAM J. Numer. Anal., 42(2) (2004), pp. 800-825.

[5] Guillaume Bal, Albert Fannjiang, George Papanicolaou and LeOnid RyzhiK, Radiative transport in a periodic structure, J. Stat. Phys., 95(1-2) (1999), pp. 479-494.

[6] Guillaume Bal, TOMASZ KOMOROWSKI AND LENYA RYZHIK, Asymptotics of the solutions of the random schrödinger equation, Arch. Ration. Mech. An., 200(2) (2011), pp. 613-664.

[7] Guillaume Bal AND Olivier Pinaud, Accuracy of transport models for waves in random media, Wave Motion, 43(7) (2006), pp. 561-578.

[8] WeIZHU BAO, SHI JIN AND PETER A MARKOWICH, On time-splitting spectral approximations for the schrödinger equation in the semiclassical regime, J. Comput. Phys., 175(2) (2002), pp. 487524. 
[9] Claudio Canuto, M Youssuff Hussaini, Alfio Quarteroni and Thomas A ZanG, Spectral Methods: Fundamentals in Single Domains, Springer-Verlag, 2006.

[10] James Colliander, Markus Keel, Gigiola Staffilani, Hideo TakaOKa and TerENCE TAO, Global well-posedness and scattering for the energy-critical schrödinger equation in $\mathbb{R}^{3}$, Ann. Math., 167(3) (2008), pp. 767-865.

[11] James Colliander, Markus Keel, Gigliola Staffilani, Hideo TaKaOKa and TerENCE TAO, A refined global well-posedness result for schrödinger equations with derivative, SIAM J. Math. Anal., 34(1) (2002), pp. 64-86.

[12] Daniel S Fisher, MAtThew PA Fisher AND DAVID A Huse, Thermal fluctuations, quenched disorder, phase transitions and transport in type-ii superconductors, Phys. Rev. B, 43(1) (1991), pp. 130-159.

[13] Daniel S Fisher And David A Huse, Directed paths in a random potential, Phys. Rev. B, 43(13) (1991), pp. 10728-10742.

[14] Jean-Pierre Fouque, Josselin Garnier, George Papanicolaou and Knut Sølna, Wave Propagation and Time Reversal in Randomly Layered Media, vol. 56, Springer Science \& Business Media, 2007.

[15] BERTRAND I HALPERIN, Green's functions for a particle in a one-dimensional random potential, Phys. Rev., 139(1A) (1965), pp. A104-A117.

[16] JINGWEI HU, SHI JIN AND DONGBIN XIU, A stochastic galerkin method for hamilton-jacobi equations with uncertainty, SIAM J. Sci. Comput., 37(5) (2015), pp. A2246-A2269.

[17] AlEXANDRU D IONESCU, Well-posedness and local smoothing of solutions of schrödinger equations, Math. Res. Lett., 12(2) (2005), pp. 193-206.

[18] SHI JIN AND ZHENG MA, The discrete stochastic galerkin method for hyperbolic equations with non-smooth and random coefficients, J. Sci. Comput., 74(1) (2018), pp. 97-121.

[19] Shi Jin, Peter MARKOWICH AND Christof Sparber, Mathematical and computational methods for semiclassical schrödinger equations, Acta Numer., 20 (2011), pp. 121-209.

[20] ShI Jin, DOngbin XIU AND XUeYu Zhu, Asymptotic-preserving methods for hyperbolic and transport equations with random inputs and diffusive scalings, J. Comput. Phys., 289 (2015), pp. $35-52$.

[21] Duane D Johnson, DM Nicholson, FJ Pinski, BL GyORfFy AND GM StOcKs, Densityfunctional theory for random alloys: Total energy within the coherent-potential approximation, Phys. Rev. Lett., 56(19) (1986), pp. pp. 2088-2091.

[22] WERNER KIRSCH, Random Schrödinger Operators a Course, Schrödinger Operators, Springer, 1989, pp. 264-370.

[23] WERNER KIRSCH AND FABIO MARTINELli, On the density of states of schrödinger operators with a random potential, J. Phys. A Math. Gen., 15(7) (1982), pp. 2139-2156.

[24] WERNER KIRSCH AND FABIO MARTINELLI, On the spectrum of schrödinger operators with a random potential, Commun. Math. Phys., 85(3) (1982), pp. 329-350.

[25] Hajo Leschke, Peter Müller and SimOne Warzel, A Survey of Rigorous Results on Random Schrödinger Operators for Amorphous Solids, Interacting Stochastic Systems, Springer, 2005, pp. 119-151.

[26] JANI LUKKARINEN AND HERBERT SPOHN, Weakly nonlinear schrödinger equation with random initial data, Invent. Math., 183(1) (2011), pp. 79-188.

[27] Mohammad Motamed, Fabio Nobile And Raúl Tempone, Analysis and computation of the elastic wave equation with random coefficients, Comput. Math. Appl., 70(10), pp. (2015), pp. 2454-2473.

[28] AKIL NARAYAN AND TAO ZHOU, Stochastic collocation methods on unstructured meshes, Com- 
mun. Comput. Phys., 18 (2015), pp. 1-36.

[29] FABIo Nobile AND RaÚl TemPONe, Analysis and implementation issues for the numerical approximation of parabolic equations with random coefficients, J. Numer. Meth. Eng. 80(6-7) (2009), pp. 979-1006.

[30] Giacomo Roati, Chiara D'Errico, Leonardo Fallani, Marco Fattori, Chiara Fort, Matteo Zaccanti, Giovanni Modugno, Michele Modugno and MasSIMO INGUSCIO, Anderson localization of a non-interacting bose-einstein condensate, Nature, 453(7197), (2008), pp. 895-898.

[31] Ruiwen SHU, JingWei Hu AND SHI Jin, A stochastic galerkin method for the boltzmann equation with multi-dimensional random inputs using sparse wavelet bases, Numer. Math. Theor. Meth. Appl., 10(2) (2017), pp. 465-488.

[32] TAO TANG AND TAO ZHOU, Convergence analysis for stochastic collocation methods to scalar hyperbolic equations with a random wave speed, Commun. Comput. Phys., 8(1) (2010), pp. 226248.

[33] Zhizhang Wu AND ZHONGYi HuANG, A bloch decomposition-based stochastic galerkin method for quantum dynamics with a random external potential, J. Comput. Phys., 317 (2016), pp. 257-275.

[34] DONGBIN XIU, Efficient collocational approach for parametric uncertainty analysis, Commun. Comput. Phys., 2(2) (2007), pp. 293-309.

[35] DongBIN XIU, Fast numerical methods for stochastic computations: a review, Commun. Comput. Phys., 5(2-4) (2009), pp. 242-272.

[36] DongBIN XIU, Numerical Methods for Stochastic Computations: a Spectral Method Approach, Princeton University Press, 2010.

[37] DongBin XIU AND JAN S Hesthaven, High-order collocation methods for differential equations with random inputs, SIAM J. Sci. Comput., 27(3) (2005), pp. 1118-1139.

[38] Dongbin XIU AND GeORge EM KARNIADAKIS, The wiener-askey polynomial chaos for stochastic differential equations, SIAM J. Sci. Comput., 24(2) (2002), pp. 619-644.

[39] KENJI YAJIMA, Existence of solutions for schrödinger evolution equations, Commun. Math. Phys., 110(3) (1987), pp. 415-426.

[40] LIANG YAN AND TAO ZHOU, Adaptive multi-fidelity polynomial chaos approach to bayesian inference in inverse problems, J. Comput. Phys., 381 (2019), pp. 110-128.

[41] GUANNAN ZHANG AND MAX GUNZBURGER, Error analysis of a stochastic collocation method for parabolic partial differential equations with random input data, SIAM J. Numer. Anal., 50(4) (2012), pp. 1922-1940. 